\title{
Las variaciones de la satisfacción vital según edad y clima organizacional en trabajadores de la salud*
}

\section{Variations in the Life Satisfaction according to the Age and Organizational Climate among the Health Workers}

\section{As variações da satisfação vital segundo idade e clima organizacional em trabalhadores da saúde}

Fecha de recepción: 12 de junio de 2017. Fecha de aprobación: 30 de agosto de 2017.

Fecha de publicación: 27 de mayo de 2019

DOI: https://doi.org/10.11144/Javeriana.rgps18-36.vsve

Rodolfo Mendoza-Llanos

Universidad del Bío-Bío, Chile

ORCID: 0000-0001-9529-570X

Emilio Moyano-Díaz

Universidad de Talca, Chile

ORCID: 0000-003-2433-9271

Cómo citar este artículo: Mendoza-Llanos R, Moyano-Díaz E. Las variaciones de la satisfacción vital según edad y clima organizacional en trabajadores de la salud. Revista Gerencia y Políticas de Salud. 2019;18(36). https://doi.org/10.11144/Javeriana.rgps18-36.vsve.

Artículo de investigación

Esta investigación fue financiada por la Universidad del Bío-Bío, Proyecto DIUBB 167824 2/I (2016-2018), otorgado al primer autor.

Autor de correspondencia. Correo electrónico: emoyano@utalca.cl 


\section{Resumen}

La satisfacción vital es un predictor de enfermedades físicas que tiene una relación consistente con la satisfacción laboral y con la edad, pero su relación con esta última es aún contradictoria. Se evalúa aquí el impacto del clima organizacional y la satisfacción laboral en la satisfacción vital de 623 trabajadores de un hospital del sur de Chile, según edad (20-29; 30-39; 40-49; 50-59 años), mediante un modelo de ecuaciones estructurales. Se observa que el clima organizacional, mediado por la satisfacción laboral, tiene un impacto directo y positivo sobre la satisfacción de vida de los trabajadores, incrementándola conforme aumenta la edad, principalmente entre los 40 y 59 años. La gestión que busque optimizar el clima organizacional así como la satisfacción laboral contribuirá a aumentar el bienestar de los trabajadores en su espacio laboral, y por transferencia también su satisfacción con la vida en general.

Palabras clave: satisfacción laboral, satisfacción personal, grupos de edad, personal de salud

\section{Abstract}

Life satisfaction is a predictor of physical diseases with a consistent relation to the age and labor satisfaction, though the relation to the age is still controversial. This work evaluates the impact of the organizational climate and the labor satisfaction on the life satisfaction of 623 workers at a hospital in southern Chile according to their age (20-29; 30-39; 40-49; 50-59 years old). It is done based on a model of structural equations. It was found that the organizational climate -as mediated by the labor satisfaction- has a direct and positive impact on the life satisfaction of health workers, which enhances as they get older and mostly in the range from 40 to 59 years old. A management intended to optimize the organizational climate as well as the labor satisfaction will contribute to enhance the health worker's well-being at the working site and, consequently, in his/her life satisfaction in general.

Keywords: labor satisfaction, personal satisfaction, age groups, health-working personnel

\section{Resumo}

A satisfação vital é um preditor de doença física que tem relação consistente com a satisfação laboral e com a idade, mas a sua relação com esta última ainda é contraditória. Avalia-se aqui o impacto do clima organizacional e a satisfação laboral na satisfação vital de 623 trabalhadores em um hospital do sul do Chile, segundo idade (20-29; 30-39; 40-49; 50-59 anos), mediante modelo de equações estruturais. Observa-se que o clima organizacional, mediado pela satisfação laboral, tem impacto direito e positivo sobre a satisfação de vida dos trabalhadores, incrementando-a conforme aumenta a idade, principalmente entre 40 e 59 anos. A gestão que vise otimizar o clima organizacional bem como a satisfação laboral contribuirá para aumentar o bem-estar dos trabalhadores no seu espaço laboral, e por transferência mesmo sua satisfação com a vida em geral.

Palavras chave: satisfação laboral, satisfação pessoal, grupos etários, pessoal de saúde 


\section{Introducción}

El actual contexto laboral chileno se caracteriza por una tendencia al envejecimiento de la fuerza laboral (1), consistente con la evolución demográfica general del país y de otros países del mundo, particularmente los europeos (2). Esto, sumado a que las demandas, motivaciones y compromisos de los empleados han cambiado en las pasadas décadas, lleva a las empresas a revisar sus modelos organizacionales. Parece necesario atender los intereses de los distintos grupos de empleados según sus diferencias, pues estos se comportarán de manera distinta dependiendo de la generación a la que pertenecen (3).

El envejecimiento de la fuerza de trabajo hace necesario generar empleos sostenibles, es decir, con la propiedad de mantener y posibilitar la permanencia en ellos de los trabajadores, ahora y en el futuro (4). Estudiar las diferencias y particularmente las relativas a la edad es considerado un tema de investigación relevante para informar a la teoría y la gestión de las organizaciones con respecto a los trabajadores en sus distintas edades y, particularmente, en la etapa de la mitad de la carrera, que a menudo es descuidada al privilegiarse la investigación sobre el trabajo y el envejecimiento (5).

Existen al menos tres aproximaciones teóricas que pueden contribuir a la comprensión del efecto de las demandas o necesidades de los diferentes grupos de empleados según su edad: la teoría del rol, el envejecimiento exitoso y el modelo de transferencia. La teoría del rol formula que los trabajadores de edad media presentan mayores demandas laborales y familiares, mayor conflicto trabajo-familia y menos apoyo social en el trabajo que los trabajadores jóvenes y viejos (6). El modelo de envejecimiento exitoso en el trabajo afirma que los trabajadores a medida que avanzan en edad mantienen o aun mejoran sus resultados en este, tales como la motivación, el bienestar y la satisfacción con la vida en el trabajo (5). El modelo de transferencia a su vez sostiene que la satisfacción en un dominio de la vida se extiende a otros dominios de vida, con una asociación positiva entre ellos (7). Estas tres perspectivas permiten explicaciones complementarias entre sí, al indicar que la percepción de la experiencia laboral dependerá de las diferencias de edad y su asociación con roles en la sociedad. 


\section{Relaciones entre edad, trabajo y satisfacción con la vida (SV)}

Sirgy reportó que los empleados mayores experimentan un mayor bienestar específico en el trabajo que los empleados más jóvenes (8). Zacher y Schmitt revisaron dieciséis estudios acerca de las características del trabajo, el bienestar ocupacional y la edad, y reportaron dos estudios relativos a las características del trabajo como mediadoras de asociación entre edad y bienestar ocupacional. Las relaciones reportadas indican que existe una relación lineal y positiva entre edad y satisfacción laboral, y que la presión del tiempo y apoyo de pares media una relación curvilínea entre edad y satisfacción laboral (5).

Desde la teoría del envejecimiento, se ha reportado que la variable clima organizacional para el envejecimiento exitoso es una dimensión que no tiene un efecto moderador entre la edad y la satisfacción laboral (9). A su vez, el clima organizacional y la satisfacción laboral aparecen como variables relevantes por sus influencias sobre el bienestar en sus distintos aspectos $(10,11)$; existe evidencia de que en servicios hospitalarios públicos se encuentra un mejor clima organizacional entre trabajadores de mayor edad (12). Así, y más generalmente, diversos estudios han dado cuenta de que el bienestar y más particularmente la satisfacción con la vida de las personas se asocian con variables propias del trabajo (13-17).

Sin embargo, no hay acuerdo en que estas variables laborales impacten por igual a los trabajadores, o según su edad, pues solo se encuentran modelos respecto de la satisfacción con la vida (18-20) e impacto del clima organizacional en la satisfacción laboral $(5,10)$.

La relación entre las variables SL, SV y edad ha sido estudiada previamente en distintas partes del mundo, pues se considera que es un tema relevante dadas las dificultades de gestión asociadas a la satisfacción laboral (10,21-26).

Shirom, Toker, Melamed, Berliner y Shapira plantean que si bien existe una estrecha relación entre satisfacción laboral y satisfacción vital, solo la satisfacción vital —en una relación negativa — tiene capacidad para predecir la incidencia de diabetes (16), siendo así un factor protector frente a esta enfermedad, tan prevalente en población adulta y mayor (27). Así, la falta 
de claridad respecto de la relación entre satisfacción laboral, satisfacción con la vida y edad dificulta la tarea de identificar en la práctica profesional la satisfacción de los distintos grupos de trabajadores e implementar planes de calidad de vida diferenciados que impacten positivamente su bienestar.

Desde un punto de vista teórico y práctico, dilucidar estas relaciones permitiría aportar evidencia más precisa para fundamentar la relevancia de manipular algunos factores organizacionales y generar impactos sobre la experiencia asociada al trabajo según edad, y con ello el establecimiento de planes y políticas dentro de las instituciones públicas de salud para mejorar la calidad de vida laboral de los trabajadores (28). Hay alguna evidencia que la implementación de políticas de desarrollo de recursos humanos genera percepción positiva del clima de desarrollo, fomenta un contrato psicológico positivo con la organización y está positivamente asociada a la motivación de continuar trabajando en esta $(29,30)$. Así, las organizaciones laborales podrán apoyar capacitando en el uso de diferentes mecanismos de afrontamiento a sus trabajadores, teniendo en consideración las diferencias en la gestión para el óptimo desempeño y el logro de metas en instituciones de salud (31).

En el presente estudio el objetivo es determinar el impacto que tiene el clima organizacional y la satisfacción laboral en la satisfacción vital de trabajadores de la salud, controlando sus edades. Como objetivos específicos se busca: (1) determinar el grado de relación entre clima organizacional (CO), satisfacción laboral (SL) y el nivel de satisfacción con la vida (SV); (2) determinar eventuales diferencias entre los distintos grupos de edad respecto de la satisfacción con la vida (SV); y (3) determinar la validez del modelo explicativo del efecto de las variables $\mathrm{CO}$ y SL sobre SV que sigue a continuación.

El modelo hipotetizado (figura 1) tiene su fundamento empírico en los reportes ya referidos, los cuales indican que la satisfacción laboral es una variable mediadora entre los elementos individuales y situacionales, ya que presenta elementos intrínsecos y extrínsecos, respectivamente $(10,32)$, siendo el clima organizacional una variable que está relacionada directa y positivamente con la satisfacción laboral de los trabajadores $(10,11)$. 


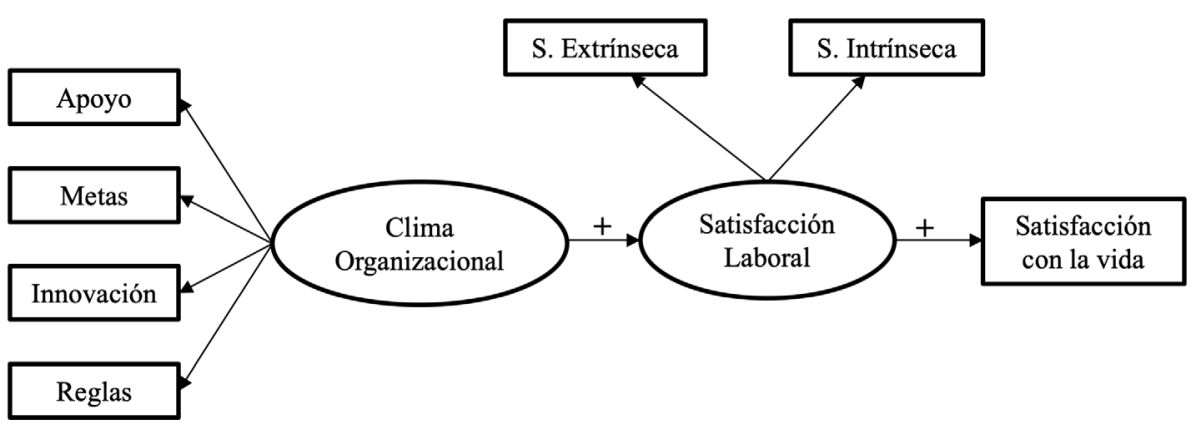

Figura 1. Modelo de relaciones hipotetizadas en este estudio

Fuente: elaboración propia

\section{Método}

El estudio es cuantitativo, transversal y no experimental. Se eligió para su realización un hospital público de alta complejidad (con alrededor de 1250 funcionarios) que atiende población general de una vasta región del sur de Chile (Bío Bío), donde trabajan empleados de distintos estamentos, áreas clínicas y administrativas, con sus correspondientes y representativas variaciones de niveles educacionales y económicos del país (10).

La muestra fue estructurada mediante el listado de personal y se extrajeron alfabéticamente los nombres correspondientes siguiendo los números impares de la lista. Quedó conformada en un 69,3\% por mujeres. La edad promedio fue 37,85 años $(\mathrm{DE}=10,95 ;$ mínimo $=20$; y máximo $=59)$, donde el 30,0\% tenía entre 20 y 29 años; el $28,1 \%$ entre 30 y 39 años; el $21,3 \%$ entre 40 y 49 años; y el 20,5\% entre 50 y 59 años. Respecto del estamento, el 10,0\% fueron auxiliares, $13,6 \%$ personal administrativo, $45,6 \%$ personal técnico, 27,4\% profesionales no médicos y 3,3\% médicos.

\section{Instrumentos}

Clima organizacional: el cuestionario FOCUS-93, compuesto por doce ítems en formato de respuesta Likert de cinco puntos (dónde 1 significa "Muy en desacuerdo" y 5 "Muy de acuerdo"), para medir cuatro dimensiones del clima organizacional: apoyo, metas, innovación y reglas. Los puntajes para cada dimensión son obtenidos del promedio de los tres ítems que componen cada una, en un rango posible de 1 (mínimo) a 15 (máximo). 
Satisfacción laboral: escala de satisfacción laboral de Warr, Cook y Wall (32), basada en la teoría de los dos factores de Herzberg referidos a las condiciones de trabajo y particularmente a los satisfactores intrínsecos y a los extrínsecos. Consta de quince reactivos en formato de respuesta tipo Likert de siete puntos, donde 7 significa "Muy satisfecho" y 1 significa "Muy insatisfecho". Los puntajes de cada dimensión corresponden a la sumatoria de los ítems que la componen, de 7 a 49 puntos como valores mínimo y máximo para satisfacción intrínseca, y de 8 a 56 puntos respectivamente para satisfacción extrínseca.

Satisfacción vital: escala de satisfacción vital de Diener, Emmons, Larsen y Griffin (28), de cinco ítems, con formato tipo Likert de siete puntos, donde 7 significa "Muy satisfecho" y 1 significa "Muy insatisfecho". Su puntuación total es la suma del puntaje obtenido para cada uno de los ítems, de modo que los puntajes teóricos mínimo y máximo corresponden a 5 y 35 puntos, respectivamente.

\section{Procedimiento}

Se constituyeron cuatro grupos de edad en tramos de 10 años: 20-29, 30-39, 40-49 y 50-59. Se realizó un análisis de confiabilidad, otro de estadísticos descriptivos generales, y de correlaciones de Pearson entre las dimensiones de SL y CO con la SV y edad; seguido por ANOVA para las variables SV y las dimensiones de la SL. Fue analizada la relación entre las dimensiones de CO, SL y su eventual impacto en la SV de los trabajadores mediante un modelo confirmatorio de ecuaciones estructurales.

El método de estimación utilizado es el de máxima verosimilitud (33). La evaluación de la bondad de ajuste de un modelo estructural se realiza mediante índices de ajuste global del modelo, se utiliza el estadístico chi²; el índice de ajuste global (GFI), que se interpreta como $\mathrm{R}^{2}$ en regresiones $\mathrm{y}$ que es válido en estimaciones mediante el método de máxima verosimilitud, considerándose un ajuste bueno sobre 0,90; el índice error de aproximación cuadrático medio (RMSEA) permite analizar los residuos, donde los valores que suponen un buen ajuste en términos de residuos son aquellos menores a 0,08. Se utilizan también los indicadores: índice de ajuste comparativo (CFI) que corresponde a una estandarización del índice de Bentler y es recomendable de usar en lugar de $\mathrm{chi}^{2}$, junto con RMSEA para muestras sobre cien casos. Se considera un buen ajuste a valores sobre 0,90 ; índice de 
ajuste no normado o índice de Tucker Lewis (TLI), para el cual se esperan valores iguales o superiores a 0,90 (34). Estos dos últimos indicadores deberían ser, a criterio de Herrero, iguales o superiores a 0,95 (35). Por tanto, los criterios de evaluación de la bondad de ajuste del modelo fueron $\mathrm{chi}^{2} / \mathrm{gl}$ $\leq \leq 3$; RMSEA $<0,08$; GFI, CFI y TLI $\geq \geq 0,95(34,35)$.

Los datos fueron procesados con el programa SPSS v. 21 y analizados con AMOS v. 21.0. y JASP v. 0.8.1.2 (36).

\section{Resultados}

De acuerdo con los resultados reportados en la tabla 1, los tres instrumentos utilizados tienen una confiabilidad adecuada, $\alpha>0,80$. Los resultados obtenidos en los rangos de edades parecen homogéneos respecto de los resultados generales.

Tabla 1. Medias y desviaciones estándar, general y por rangos de edad, y confiabilidades para los tres instrumentos

\begin{tabular}{cccccccc}
\multicolumn{7}{c}{ Satisfacción laboral } & \multicolumn{7}{c}{ Clima organizacional } \\
\hline \multicolumn{1}{c}{ Edad } & SV & SI & SE & A & M & I & R \\
\hline General & $25,27(6,49)$ & $33,49(8,76)$ & $37,97(9,23)$ & $3,73(1,04)$ & $3,75(1,01)$ & $3,59(0,848)$ & $3,75(0,84)$ \\
\hline $20-29$ & $26,34(5,98)$ & $35,53(8.23)$ & $39,69(8,78)$ & $3,96(0,94)$ & $4,01(0,88)$ & $3,66(0,78)$ & $3,77(0,79)$ \\
\hline $30-39$ & $24,81(6,57)$ & $32,22(8,64)$ & $36,51(9,25)$ & $3,61(1,04)$ & $3,60(1,00)$ & $3,55(0,78)$ & $3,72(0,79)$ \\
\hline $40-49$ & $24,80(6,88)$ & $32,24(8,11)$ & $37,13(8,90)$ & $3,60(1,10)$ & $3,58(1,07)$ & $3,46(0,94)$ & $3,82(0,85)$ \\
\hline $50-59$ & $24,81(6,56)$ & $33,52(9,79)$ & $38,31(9,85)$ & $3,69(1,08)$ & $3,76(1,05)$ & $3,68(0,90)$ & $3,70(0,94)$ \\
\hline $\begin{array}{l}\alpha \text { de } \\
\text { Cronbach }\end{array}$ & 0,879 & 0,878 & 0,833 & 0,835 & 0,853 & 0,845 & 0,804 \\
\hline \begin{tabular}{l} 
N items \\
\hline
\end{tabular} & 5 & 7 & 8 & 3 & 3 & 3 & 3 \\
\hline
\end{tabular}

* SV = satisfacción vital; SI = satisfacción intrínseca; $\mathrm{SE}=$ satisfacción extrínseca; $\mathrm{A}=$ apoyo; $\mathrm{M}=$ metas; I = innovación; $R$ = reglas.

Fuente: elaboración propia 
Las correlaciones (tabla 2) entre edad con las otras variables son: satisfacción $\operatorname{vital}(r=-, 070 ; p=0,081)$; satisfacción laboral intrínseca $(r=-, 089 ; p=0,027)$; satisfacción laboral extrínseca $(r=-, 053 ; p=, 183)$; apoyo $(r=-, 090 ; p=, 025)$ y metas $(r=-, 096 ; p=0,016)$; innovación $(r=-, 025 ; p=0,528)$, y reglas $(r=-, 031$; $p=0,437)$. Estos resultados pueden enmascarar otras relaciones entre las variables, pues existe evidencia que indica que la SV varía con la edad (37).

Tabla 2. Correlaciones entre satisfacción vital y las dimensiones de satisfacción laboral y clima organizacional

\begin{tabular}{|c|c|c|c|c|c|c|c|c|c|}
\hline & & \multirow[b]{2}{*}{$\mathbf{N}$} & & \multicolumn{2}{|c|}{ Satisfacción laboral } & \multicolumn{2}{|c|}{ Clima organizacional } & \multirow[b]{2}{*}{ I } & \multirow[b]{2}{*}{$\mathbf{R}$} \\
\hline & & & & SI & SE & A & M & & \\
\hline & & 623 & Edad & $-0,089^{\star \star \star}$ & $-0,053^{\star \star \star}$ & $-0,090^{\star \star \star}$ & $-0,096^{\star \star \star}$ & $-0,025^{\star \star \star}$ & $-0,031^{\star \star \star}$ \\
\hline & & 623 & SV & $0,358^{\star \star *}$ & $0,365^{\star \star \star}$ & $0,278^{\star \star \star}$ & $0,232^{\star \star \star}$ & $0,193^{\star * \star}$ & $0,059^{\star \star \star}$ \\
\hline \multirow{6}{*}{ 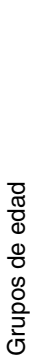 } & $20-29$ & 187 & SV & $0,281^{\star \star \star}$ & $0,296^{\star \star \star}$ & $0,181^{\star \star \star}$ & $0,110^{\star \star \star}$ & $0,146^{\star \star \star}$ & $-0,016^{\star \star *}$ \\
\hline & $30-39$ & 175 & SV & $0,228^{\star * *}$ & $0,234^{\star \star \star}$ & $0,193^{\star \star \star}$ & $0,161^{\star \star \star}$ & $0,026^{\star *}$ & $-0,063^{\star \star \star}$ \\
\hline & $40-49$ & 133 & SV & $0,445^{\star \star \star}$ & $0,434^{\star \star \star}$ & $0,339^{\star * *}$ & $0,271^{* \star *}$ & $0,286^{\star \star *}$ & $0,273^{\star \star \star}$ \\
\hline & $50-59$ & 128 & SV & $0,486^{\star \star \star}$ & $0,517^{\star \star \star}$ & $0,384^{\star \star *}$ & $0,358^{\star * \star}$ & $0,327^{\star \star \star}$ & $0,069^{\star \star \star}$ \\
\hline & $20-39$ & 362 & SV & $0,270^{\star \star \star}$ & $0,279^{\star \star \star}$ & $0,205^{\star \star \star}$ & $0,159^{\star \star \star}$ & $0,093^{\star \star *}$ & $-0,036^{\star \star \star}$ \\
\hline & $40-59$ & 261 & SV & $0,462^{\star \star \star}$ & $0,474^{\star \star \star}$ & $0,360^{\star \star *}$ & $0,311^{\star \star \star}$ & $0,303^{\star \star \star}$ & $0,169^{\star \star \star}$ \\
\hline
\end{tabular}

${ }^{*} \mathrm{p}<0,05,{ }^{* *} \mathrm{p}<0,01,{ }^{* \star *} \mathrm{p}<0,001 ; \mathrm{SI}=$ satisfacción intrínseca; SE = satisfacción extrínseca; $\mathrm{A}=$ apoyo; $M=$ metas; I = innovación; $R$ = reglas. Fuente: elaboración propia.

Fuente: elaboración propia

\section{Comparaciones de promedios}

Al comparar los grupos de acuerdo con edad (véase figura 2), se observa diferencias en el nivel general de satisfacción laboral $\left(F_{(3,619)}=5,069 ; p=\right.$ 0,$\left.002 ; \eta^{2}=0,024\right)$. El análisis post-hoc arroja que las diferencias son estadísticamente significativas solo entre las personas de 20-29 y 30-39 años $(t=3,569 ; p=0,002 ; d=0,383), 40-49(t=2,983 ; p=0,016 ; d=0,356)$, respectivamente. Al comparar los grupos según edad, se advierten diferencias en satisfacción extrínseca $\left(F_{(3,619)}=4,103 ; p=0,007 ; \eta^{2}=0,019\right)$, pero solo entre los grupos de 20-29 y 30-39 años ( $t=3,298 ; p=0,006 ; d=0,353)$. 

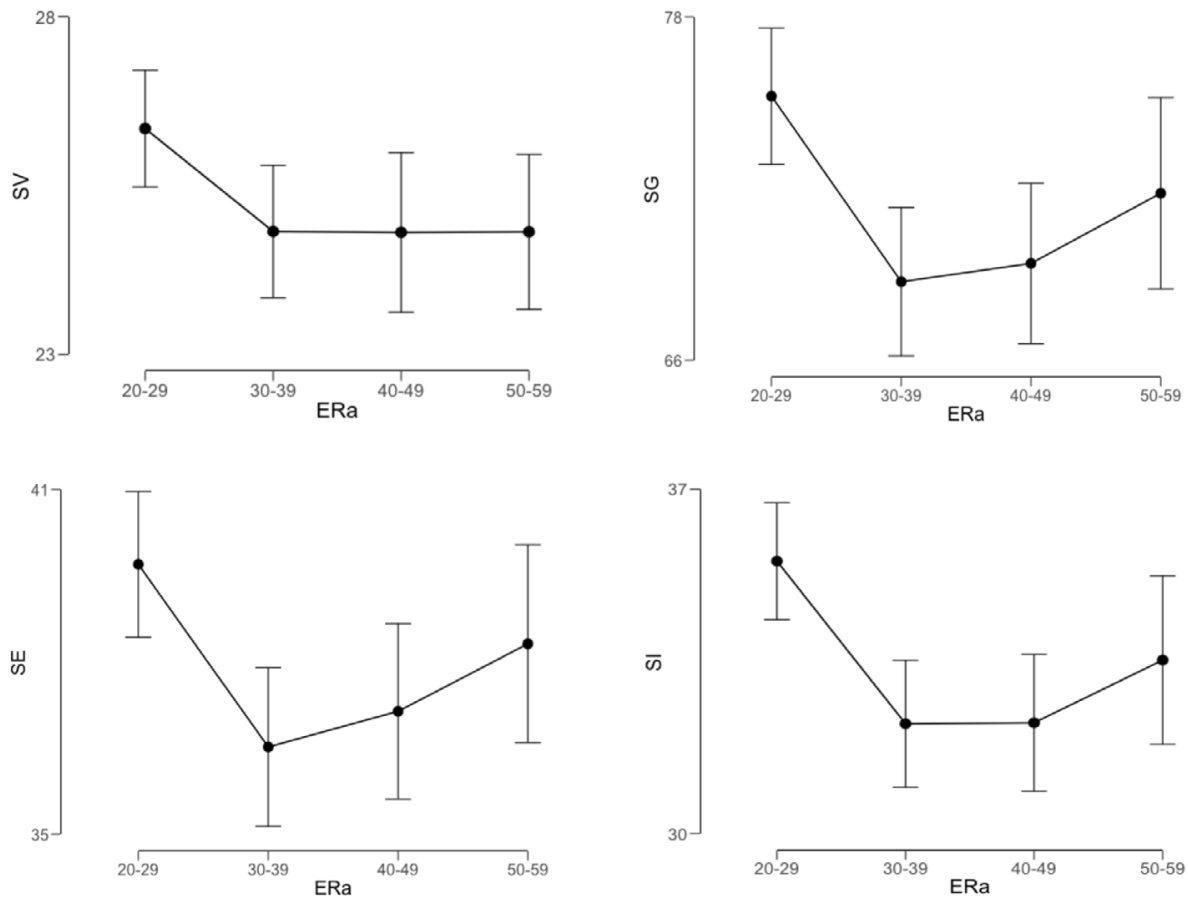

Figura 2. Medias e intervalos de confianza para niveles de satisfacción vital y laboral general según rangos de edad

* SV = satisfacción vital; SG = satisfacción laboral; SI = satisfacción intrínseca; SE = satisfacción extrínseca; $E R a=$ edad por rangos.

Fuente: elaboración propia

Al comparar los grupos de acuerdo con edad, se observan diferencias en satisfacción intrínseca $\left(F_{(3,619)}=5,621 ; p<0,001 ; \eta^{2}=0,027\right)$, específicamente entre los grupos de 20-29 y 30-39 ( $t=3,628 ; p=0,002 ; d=0,392)$ y 40-49 años ( $t=3,344 ; p=0,005 ; d=0,402)$. Al comparar los grupos de acuerdo con edad, no se observan diferencias en satisfacción con la vida $\left(F_{(3,619)}=2,440 ; p=0,063\right)$ ni por estamentos $\left(F_{(6,616)}=2,101 ; p=0,051\right)$.

\section{Modelo estructural}

Para ajustar el modelo se requirió una reespecificación fijando una correlación entre los indicadores de metas y reglas, de acuerdo con el modelo teórico de valores en competencias (38), puesto que se trata de dos dimensiones dirigidas a orientar y regular el comportamiento de las personas. 
Tabla 3. Índices de ajuste para los modelos para cada rango de edad

\begin{tabular}{llllll}
\hline Índices & Valor óptimo & $\mathbf{2 0 - 2 9}$ & $\mathbf{3 0 - 3 9}$ & $\mathbf{4 0 - 4 9}$ & $\mathbf{5 0 - 5 9}$ \\
\hline$\chi^{2} / \mathrm{gl}$ & $<3$ & 2,051 & 2,306 & 2,018 & 1,701 \\
\hline GFI & $>0,95$ & 0,963 & 0,957 & 0,951 & 0,959 \\
\hline RMSEA & $0,05-0,08$ & 0,075 & 0,087 & 0,088 & 0,074 \\
\hline CFI & $>0,95$ & 0,980 & 0,971 & 0,977 & 0,984 \\
\hline TLI & $>0,95$ & 0,966 & 0,948 & 0,959 & 0,972 \\
\hline
\end{tabular}

GFI = índice de ajuste global; RMSEA = índice error de aproximación cuadrático medio; $\mathrm{CFI}$ = índice de ajuste comparativo; TLI = índice de ajuste no normado.

Fuente: elaboración propia

El modelo permite explicar la varianza de la satisfacción vital, de manera distinta de acuerdo con los grupos de edades: 20-29 años $\left(R^{2}=0,09\right)$; 30-39 años $\left(R^{2}=0,06\right) ; 40-49$ años $\left(R^{2}=0,22\right) ; 50-59$ años $\left(R^{2}=0,28\right)$. Así también, los tamaños de los efectos de acuerdo con el modelo hipotético planteado indican que la satisfacción laboral tiene un impacto mayor en la satisfacción vital que el que tiene el clima organizacional sobre la satisfacción laboral.

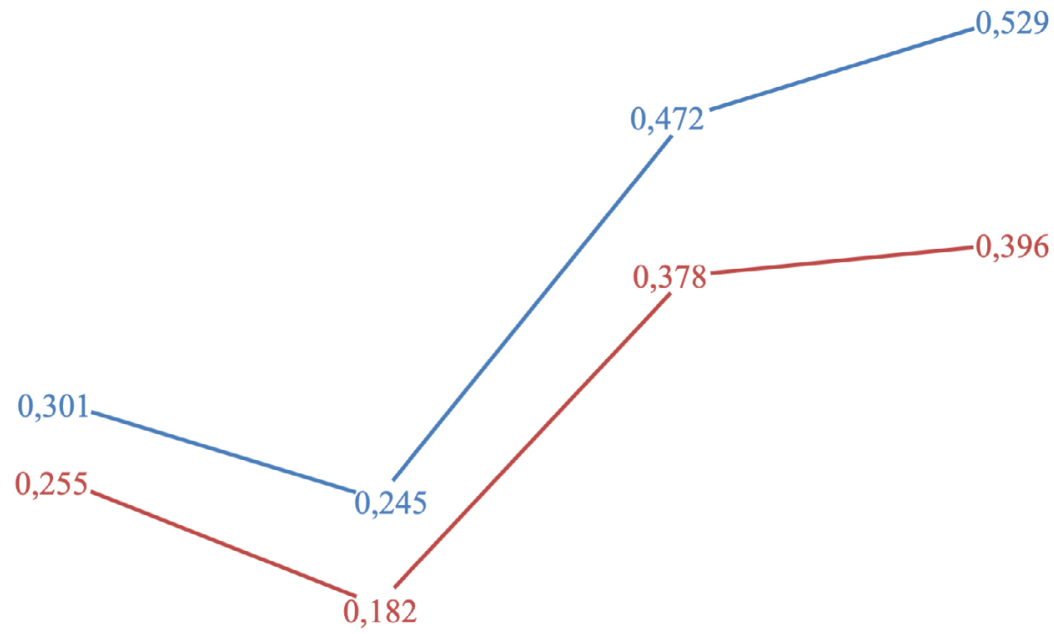

$$
\begin{array}{cccc}
\text { 20-29 años } & \text { 30-39 años } & 40-49 \text { años } & 50-59 \text { años } \\
& - \text {-Satisfacción Laboral } & - & \text { - Clima organizacional }
\end{array}
$$

Figura 3. Efectos estandarizados del clima organizacional y la satisfacción laboral sobre la satisfacción vital según cuatro tramos de edad

Fuente: elaboración propia 
En términos generales, se confirman las relaciones hipotetizadas entre clima organizacional y satisfacción laboral, y entre esta última y satisfacción vital, siendo positivas y estadísticamente significativas, pero los efectos varían de manera significativa de acuerdo con la edad (figura 3). Así, se observa una clara progresión en la que conforme aumenta la edad se incrementa también la satisfacción laboral y el clima organizacional en su impacto sobre la satisfacción vital, con la excepción del segundo tramo de edad -30 a 39 años- que es donde se ubican los indicadores más bajos de impacto de ambas variables.

\section{Discusión}

Globalmente, se observa que a medida que aumenta la edad de los trabajadores su satisfacción con la vida general se ve cada vez más positivamente afectada por el clima organizacional y por su propia satisfacción laboral. Esto puede asociarse a la existencia de diferentes etapas del desarrollo laboral. Así, por ejemplo, aquellas edades iniciales en las que los trabajadores aún no tienen una "historia" -ni menos "consolidada" - dentro del servicio donde trabajan, a diferencia de aquellas otras propias de tramos de edad más avanzada donde probablemente exista una mayor consolidación de la carrera funcionaria y una mayor identificación con el trabajo y la historia - ya compartida - del lugar. También, se puede pensar que existen ciertas motivaciones externas de tipo individual que podrían disminuir con el paso del tiempo, como por ejemplo la responsabilidad de criar y educar a hijos de corta edad, lo que los lleva a basar (o presiona) una mayor parte de su identidad o sentido de vida en el trabajo, constituyéndolo en una fuente renovada de importancia para la autoestima personal.

Los resultados apoyan la idea de que después de los cuarenta años la satisfacción laboral es causa o genera alrededor del $25 \%$ de la satisfacción vital del trabajador, lo cual es un indicador relevante. El resultado de menor impacto obtenido aquí para el grupo de 30-39 años lleva a preguntarse qué acontece o qué explica que para ellos el impacto de las variables organizacionales sea menor. Tal vez exista en esa etapa de desarrollo un foco en la maternidad-paternidad, lo cual pone el centro y energías del trabajador principalmente —o quizá exclusivamente- en el ámbito familiar más que en el laboral. Esto podría ser un tema de interés para futuras investigaciones. De cualquier manera, los resultados apoyan lo planteado por la literatura 
$(5,37,39)$ referido a que las diferencias de edad explicarían más varianza en trabajadores mayores que en los más jóvenes.

Desde el conjunto de resultados obtenidos se puede proponer la adopción de medidas prácticas para generar condiciones de trabajo que favorezcan aún más el clima organizacional y la satisfacción laboral de los trabajadores de la salud simultáneamente, ya que para todos será beneficioso, y particularmente provocando un impacto de mayor intensidad progresiva sobre la vida en general de los trabajadores de la salud conforme la edad de estos aumenta.

Por otro lado, las implicancias de mantener un buen clima organizacional en las organizaciones y el impacto indirecto que ello tiene en la satisfacción vital de los trabajadores de la salud indican que su efecto antes de los cuarenta años es menor al que tiene posteriormente, lo que subraya la relevancia de utilizar esta variable como indicador de gestión de personal en la institución y, así, favorecer la calidad de vida laboral y general de los trabajadores. El resultado claramente apoya la idea de que los ambientes en los cuales las personas desempeñan sus labores no solo tienen influencia en ese espacio particular de trabajo, sino también un impacto real e irradiado de modo amplio a los diferentes ámbitos de desarrollo de sus vidas, siendo un aporte empírico en apoyo al modelo de transferencia.

Mickel y Dallimore plantean que la etapa de la vida en que los trabajadores se encuentren contribuye a entender por qué ciertos grupos de edad afrontan diferencialmente las circunstancias vitales (40). Así, por ejemplo, sostienen que el apoyo social en el trabajo de los miembros de la familia resulta ser más efectivo para aliviar el conflicto familia-trabajo para los menores de 45 años.

Entre las limitaciones del presente estudio se encuentra que, puesto que se trata de un diseño de tipo transversal, no permite concluir causalmente respecto de los cambios relacionados con la edad, sino solo comparar grupos. Por otro lado, al evaluar únicamente aspectos del ámbito laboral, no se puede indicar que estos resultados no pudieran también estar influidos por la interacción con otros ámbitos de la esfera personal, ya que, por ejemplo, y según sostiene la literatura respecto de la satisfacción vital (16), esta incorpora diversos aspectos -incluso los físicos-, capturando elementos más integrales de la persona y ámbitos diversos de esta, no así la satisfacción laboral, que podría estar influida solo por elementos contingentes propios del trabajo. 
Una de las fortalezas de esta investigación es que el uso de ecuaciones estructurales como técnica de análisis permite confirmar empíricamente la estructura conceptual establecida a priori y conocer el papel de cada elemento en el conjunto global de esa estructura (35). En ese sentido, si bien se repiten los resultados en relación con la dirección de las relaciones entre variables, se profundiza aquí respecto del impacto que tiene la edad de los trabajadores al explicar su satisfacción con la vida.

\section{Referencias}

1. Hermosilla-Ávila A, Paravic-Klijn T, Valenzuela-Suazo S. Fuerza laboral que envejece, ¿qué hacer ante esta tendencia? Ciencia y Trabajo. 2015;17(54):166-70. Disponible en: https://scielo.conicyt.cl/pdf/cyt/v17n54/art02.pdf

2. Rodríguez V, Rodríguez Mañas L, Sancho Castiello M, Díaz Martín R. Envejecimiento. La investigación en España y Europa. Revista Española de Geriatría y Gerontología. 2012;47(4):174-9.

3. Zenteno-Hidalgo A, Durán C. Factores y prácticas de alto desempeño que influyen en el clima laboral: análisis de un caso. Innovar. 2016;26(59):119-36. doi: 10.15446/innovar.v26n59.54367.

4. Van Dam K, van Vuuren E, Kemps S. Sustainable employment: the importance of intrinsically valuable work and age-supportive climate. The International Journal of Human Resource Management. 2017;28(17):2449-72. doi: 10.1080/09585192.2015.1137607.

5. Zacher H, Schmitt A. Work characteristics and occupational well-being: the role of age. Front Psychol 2016;7:1411. doi:10.3389/fpsyg.2016.01411.

6. Huffman A, Culbertson SS, Henning JB, Goh A. Work-family conflict a cross the lifespan. J Manage Psychol. 2013;28:761-80. doi:10.1108/JMP-07-2013-0220.

7. Jiménez A, Moyano E. Factores laborales de equilibrio entre trabajo y familia: medios para mejorar la calidad de vida. Universum. 2008;23(1):116-33. doi: 10.4067/S0718-23762008000100007.

8. Sirgy M. Employee Well-being: an integrative perspective. En Reilly N, Sirgy M, Allen Gorman C, editores. Work and quality of life. Ethical practice in organizations. Dordrecht: Springer; 2012. p.35-63. doi:10.1007/978-94-0074059-4_3.

9. Zacher H, Yang J. Organizational climate for successful aging. Frontiers in Psychology. 2016;7:1-12. doi: 10.3389/fpsyg.2016.01007.

10. Mendoza R. Insatisfacción laboral como predictor del ausentismo en un hospital público. Revista Médica de Chile. 2015;143(8):1028-33. Disponible en: https:// scielo.conicyt.cl/pdf/rmc/v143n8/art10.pdf 
11. Chiang M, Salazar M, Núñez A. Clima organizacional y satisfacción laboral en un establecimiento de salud pública: Hospital tipo 1. Theoria. 2007;16(2):61-76.

12. Muñiz J, Peña-Suárez E, de la Roca Y, Fonseca-Pedrero E, Cabal AL, GarcíaCueto E. Organizational climate in Spanish public health services: administration and services staff. International Journal of Clinical and Health Psychology. 2014;14(2):101-10. doi: 10.1016/S1697-2600(14)70043-2.

13. Giacopelli NM, Simpson KM, Dalal RS, Randolph KL, Holland SJ. Maximizing as a predictor of job satisfaction and performance: A tale of three scales. Judgment and Decision Making. 2013;8(4): 448-69. Disponible en: http://journal.sjdm. org/13/13327/jdm13327.pdf

14. Polo J, Fernández M, Ramírez R. Diseño del trabajo y satisfacción con la vida. Revista Venezolana de Gerencia. 2012;17(59):466-81.

15. Hakanen J, Schaufeli W. Do burnout and work engagement predict depressive symptoms and life satisfaction? A three-wave seven-year prospective study. Journal of Affective Disorders. 2012;141 (2-3):415-24. doi: 10.1016/j.jad.2012.02.043.

16. Shirom A, Toker S, Melamed S, Berliner S, Shapira I. Life and job satisfaction as predictors of the incidence of diabetes. Applied Psychology: Health and WellBeing. 2012;4(1):31-48. doi:10.1111/j.1758-0854.2011.01054.x.

17. Ramírez M, Zurita R. Variables organizacionales y psicosociales asociadas al síndrome de burnout en trabajadores del ámbito educacional. POLIS, Revista de la Universidad Bolivariana. 2010; 9 (25):515-34. doi: 10.4067/S071865682010000100029 .

18. Kööts-Ausmees L, Realo A. The association between life satisfaction and selfreported health status in Europe. European Journal of Personality. 2015;29:64757. doi: 10.1002/per.2037.

19. Realo A, Dobewall H. Does life satisfaction change with age? A comparison of Estonia, Finland, Latvia and Sweden. Journal of Research in Personality. 2011;45:297-308. doi: 10.1016/j.jrp.2011.03.004.

20. Cuadro JL, Pérez-Roncero G, López-Baena M, Cuadros-Celorrio A, FernándezAlonso A. Satisfacción vital y factores sociodemográficos en mujeres de mediana edad. Enfermería Clínica. 2014;24(6):315-22. doi: 10.1016/j.enfcli.2014.07.003.

21. Wegge J, Schmidt KH, Parkes C, van Dick R. 'Taking a sickie': Job satisfaction and job involvement as interactive predictors of absenteeism in a public organization. Journal of Occupational and Organizational Psychology. 2007;80:77-89. doi: 10.1348/096317906X99371.

22. Cuadra A, Veloso C. Liderazgo, clima y satisfacción laboral en las organizaciones. Universum. 2007;22(2):40-56. doi: 10.4067/S0718-23762007000200004. 
23. Fernández B, Paravic T. Nivel de satisfacción laboral en enfermeras de hospitales públicos y privados de la provincia de Concepción, Chile. Ciencia y Enfermería. 2003;9(2):57-66. doi: 10.4067/S0717-95532003000200006.

24. Parra S, Paravic T. Satisfacción laboral en enfermeras/os que trabajan en el Sistema de Atención Médica de Urgencia (SAMU). Ciencia y Enfermería. 2002;8(2):37-48. doi: 10.4067/S0717-95532002000200005.

25. Goldberg C, Waldman D. Modeling employee absenteeism: testing alternative measures and mediated effect based on job satisfaction. Journal of Organizational Behavior. 2000;21:665-76. doi: org/10.1002/1099-1379(200009)21:6<665::AIDJOB48>3.0.CO;2-P.

26. Scott K, Taylor GS. An examination of conflicting findings on the relationship between job satisfaction and absenteeism: a meta-analysis. Academy of Management Journal. 1985;28(3):599-612. doi: 10.2307/256116.

27. Organización Mundial de la Salud (OMS). Diabetes. Nota descriptiva No. 312. 2012. Disponible en: http://www.who.int/mediacentre/factsheets/fs312/es/index. html

28. Moyano-Díaz E. Concepto, modelos y algunas evidencias relativas a la calidad de vida laboral. En Zúñiga-Rivas C, editor. Psicología, sociedad y equidad: aportes y desafíos. Santiago: Salesianos Impresores; 2012; p. 223-47.

29. Ke YT, Hung CH. Predictors of nurses' intent to continue working at their current hospital. Nursing Economics. 2017;35(5):259-66.

30. Polat T, Bal MP, Jansen GWP. How do development HR practices contribute to employees' motivation to continue working beyond retirement age? Work, Aging and Retirement. 2017;3(4):366-78. doi: 10.1093/workar/wax007.

31. Molina-Marím G, Oquendo-Lozano T, Rodríguez-Garzón S, Montoya-Gómez N, Vesga-Gómez C, Lagos-Campos N, Almanza-Payares R, Chavarro-Medina M, Goenaga-Jiménez E, Arboleda-Posada G. Gestión del talento humano en salud pública. Un análisis en cinco ciudades colombianas. Rev Gerenc Polít Salud. 2016;15(30):108-25. doi: http://dx.doi.org/10.11144/Javeriana.rgyps15-30.gtsp.

32. Alonso P. Diferencias en la percepción de la satisfacción laboral en una muestra de personal de administración. Boletín de Psicología. 2006;88:49-64.

33. Ximénez M, García A. Comparación de los métodos de estimación de máxima verosimilitud y mínimos cuadrados no ponderados en el análisis factorial confirmatorio mediante simulación Monte Carlo. Psicothema. 2005;17(3):528-35. Disponible en: http://www.psicothema.com/psicothema.asp? $\mathrm{id}=3140$

34. Schermelleh-Engel K, Moosbrugger H, Müller H. Evaluating the fit of structural equation models: tests of significance and descriptive goodness-of-fit measures. Methods of Psychological Research Online. 2003;8(2):23-74. 
35. Herrero J. El análisis factorial confirmatorio en el estudio de la estructura y estabilidad de los instrumentos de evaluación: un ejemplo con el cuestionario de Autoestima CA-14. Intervención Psicosocial. 2010;19(3):289-300. Disponible en: http://scielo. isciii.es/scielo.php?script $=$ sci_arttext\&pid $=$ S1132-05592010000300009

36. JASP Team. JASP. 2017 (Version 0.8.1.2) [Computer software].

37. Sarabia C. Envejecimiento exitoso y calidad de vida. Su papel en las teorías del envejecimiento. Gerokomos. 2009; 20(4):172-4. Disponible en: http://scielo.isciii. es/scielo.php?script=sci_arttext\&pid=S1134-928X2009000400005

38. Quinn RE, Rohrbaugh J. A spatial model of effectiveness criteria: towards a competing values approach to organizational analysis. Management Science. 1983; 29(3):363-77.

39. Jones MD. Which is a better predictor of job performance: Job satisfaction or life satisfaction. Journal of Behavioral and Applied Management. 2006;8(1):20-42.

40. Mickel A, Dallimore E. Work stress: help me get my feet back on the ground. En Reilly N, Sirgy M, Allen Gorman C, editores. Work and quality of life. Ethical practice in organizations. Dordrecht: Springer; 2012. p. 65-78. doi:10.1007/97894-007-4059-4_4. 